\title{
Extensively drug-resistant tuberculosis: epidemiology and management
}

This article was published in the following Dove Press journal:

Clinical Epidemiology

I April 2014

Number of times this article has been viewed

\begin{abstract}
Alberto Matteelli'
Alberto Roggi'

Anna CC Carvalho ${ }^{2}$

'Institute of Infectious and Tropical Diseases, WHO Collaborating Centre for TB/HIV Co-Infection, University of Brescia, Brescia, Italy; ${ }^{2}$ Laboratory of Innovations in Therapies, Education and Bioproducts (LITEB), Oswaldo Cruz Institute (IOC), Oswaldo Cruz Foundation (Fiocruz), Rio de Janeiro, Brazil
\end{abstract}

\begin{abstract}
The advent of antibiotics for the treatment of tuberculosis (TB) represented a major breakthrough in the fight against the disease. However, since its first use, antibiotic therapy has been associated with the emergence of resistance to drugs. The incorrect use of anti-TB drugs, either due to prescription errors, low patient compliance, or poor quality of drugs, led to the widespread emergence of Mycobacterium tuberculosis strains with an expanding spectrum of resistance. The spread of multidrug-resistant (MDR) strains (ie, strains resistant to both isoniazid and rifampicin) has represented a major threat to TB control since the 1990s. In 2006, the first cases of MDR strains with further resistance to fluoroquinolone and injectable drugs were described and named extensively drug-resistant TB (XDR-TB). The emergence of XDR-TB strains is a result of mismanagement of MDR cases, and treatment relies on drugs that are less potent and more toxic than those used to treat drug-susceptible or MDR strains. Furthermore, treatment success is lower and mortality higher than achieved in MDR-TB cases, and the number of drugs necessary in the intensive phase of treatment may be higher than the four drugs recommended for MDR-TB. Linezolid may represent a valuable drug to treat cases of XDR-TB. Delamanid, bedaquiline, and PA-824 are new anti-TB agents in the development pipeline that have the potential to enhance the cure rate of XDR-TB. The best measures to prevent new cases of XDR-TB are the correct management of MDR-TB patients, early detection, and proper treatment of existing patients with XDR-TB.
\end{abstract}

Keywords: XDR-TB, epidemiology, control, diagnosis, treatment

\section{Introduction}

The fight between humankind and Mycobacterium tuberculosis has lasted thousands of years, during which the human species could only rely on the efficiency of natural defenses of its immune system, ${ }^{1}$ thus paying a toll of $50 \%$ mortality within 5 years after the onset of the disease. ${ }^{2}$ The advent of the antibiotic era represented a striking event in this battle, and during the two decades from the discovery of streptomycin in 1943 to that of rifampicin in 1963, tuberculosis (TB) was turned from a devastating disease into a fully curable one.

In the last two decades, the World Health Organization (WHO), through its Stop TB Department, led a huge worldwide effort to curb the TB epidemic. By setting clear and effective global strategies (directly observed treatment, short course (DOTS) first, followed by the Stop TB strategy) and supporting their implementation by the majority of national TB programs in high-burden countries, it was possible to achieve the current decline in TB incidence worldwide, ${ }^{3}$ and the Millennium Development Goals were met (in the case of TB) well before 2015.,4 The development of rapid diagnostic tests for
Correspondence: Alberto Matteelli Institute of Infectious and Tropical Diseases, WHO Collaborating Centre for TB/HIV Co-Infection, University of Brescia, I Piazzale Spedali Civili, Brescia 25I23, Italy

Tel +390303995802

$\mathrm{Fax}+39030303061$

Email matteelli@med.unibs.it 
TB such as Xpert MTB/RIF ${ }^{5,6}$ and the development of new TB drugs ${ }^{7}$ are additional factors for optimism regarding the scope of TB elimination. On the other hand, the outstanding role played by the social determinants of TB and the threat represented by resistance to anti-TB drugs, in particular to second-line ones, remain unsolved and require further collaborative efforts. ${ }^{4}$

In particular, M. tuberculosis' resistance to antibiotics which had been already described at the time of the introduction of streptomycin in clinical practice, ${ }^{8}$ has developed to the point that now drug resistance represents one of the most daunting challenges to disease control worldwide. ${ }^{9,10}$

Extensively drug-resistant TB (XDR-TB) is defined as TB caused by a multidrug-resistant (MDR) strain (ie, resistant to at least rifampicin and isoniazid) that is also resistant to any fluoroquinolone (FQ) and any of the second-line injectable drugs, such as capreomycin, kanamycin, or amikacin. ${ }^{11}$ From 2006, when the first report on XDR-TB was published, ${ }^{12}$ until the end of 2012, 92 countries had reported the presence of at least one case of XDR-TB. ${ }^{3}$ The term "totally drug-resistant TB" (TDR) was proposed to define TB cases with a resistance profile beyond XDR-TB, in which the strain would be virtually resistant to all available first- and second-line drugs. ${ }^{13-16}$ However, due to limitations in availability, accuracy, and reproducibility of current drug-susceptibility methods, the adoption of a useful definition beyond the one currently used for XDR-TB is precluded. ${ }^{17}$ Moreover, the implication that a TB case is incurable, which is embraced by the term TDR, should be avoided for the sake of the patient's dignity, even considering the clear evidence that the prognosis worsens when the pattern of resistance increases.

In this review we provide an update of the main epidemiologic aspects related to XDR-TB, and focus on the measures currently available to manage the disease.

\section{Research strategy}

We searched Medline with the medical subject heading "extensively resistant tuberculosis" with the subheadings "epidemiology" and "prevention and control" for articles published between January 1, 2006 and April 30, 2013. Moreover, we looked for information on XDR-TB on the WHO, Centers for Disease Control and Prevention, and European Centers for Disease Control and Prevention websites. Additional articles (original papers and reviews) were retrieved from the list of references of manuscripts initially selected. Manuscripts written in English, French, Spanish, Italian, and Portuguese were eligible for consultation.

\section{Epidemiology of XDR-TB in the world}

In 2012, the estimated global burden of MDR-TB was 450,000, including 300,000 incident MDR-TB cases. Approximately half of the incident cases of MDR-TB were in the People's Republic of China, India, and the Russian Federation. ${ }^{3}$ Almost $4 \%$ of all new TB cases and more than $20 \%$ of those with previous history of TB treatment were estimated to be MDR-TB. However, just 94,000 MDR-TB cases were reported to the WHO in 2012, which corresponds to less than a third of the estimated cases among patients with pulmonary $\mathrm{TB}^{3}{ }^{3}$ The gap between reported and estimated cases is due to the limited access to drug-susceptibility testing (DST) for first- and second-line anti-TB drugs in many countries.

By the end of 2012, 92 countries had reported cases of XDR-TB, including 13 countries and territories that had reported more than ten XDR-TB cases in a single year. ${ }^{3}$ The average percentage of XDR-TB cases among MDR-TB cases was $9.6 \%(95 \% \mathrm{CI}: 8.1 \%-11 \%){ }^{3}$. The proportion of XDR-TB among MDR-TB cases was highest in Azerbaijan (Baku city, 12.8\%), Belarus (11.9\%), Latvia (16\%), Lithuania (24.8\%), and Tajikistan (Dushanbe city and Rudaki district, $21.0 \%){ }^{3}$

Cases with XDR-TB may be virtually untreatable, depending on the level of resistance to second-line drugs and on the efficiency of the health system in each given setting. Moreover, conditions inherent to the patient's immunity can contribute to the high rates of morbidity and mortality associated with XDR-TB. In the nosocomial outbreak described in KwaZulu-Natal in South Africa, the mortality rate among human immunodeficiency virus (HIV)-positive patients with limited or no access to highly active antiretroviral therapy was $98 \%$, after a median survival period from diagnosis of only 16 days (interquartile range $6-37$ days).${ }^{18}$ More recent studies have found mortality rates that are not significantly different between HIV-positive patients on highly active antiretroviral therapy and HIV-negative ones. ${ }^{19,20}$

\section{Risk factors for MDR- and XDR-TB}

Incorrect TB treatment is the main risk factor for the development of resistance among TB cases, and it is usually associated with intermittent drug use, errors in medical prescription, poor patient adherence, and low quality of TB drugs. ${ }^{21}$

The strongest risk factor for resistance to second-line drugs is previous and mainly incorrect use of these drugs. ${ }^{22}$ Many patients with XDR-TB report two or more previous treatment courses, and the number of previous treatment 
courses is higher among cases with XDR-TB compared with cases with MDR-TB. ${ }^{23}$ In the study of Dheda et al, $72 \%$ of XDR patients had previously been diagnosed as MDR-TB cases, confirming the role of ineffective TB programs in generating XDR-TB. ${ }^{19}$

In a retrospective cohort analysis of treatment outcomes among MDR-TB patients, in $6 \%$ of those who progressed to XDR-TB during treatment, the presence of bilateral and cavitary lesions was associated with a 3.5-fold greater hazard of developing XDR-TB. ${ }^{24}$ Prior exposure to second-line injectable drugs (hazard ratio 3.7) and each additional month in which a patient failed to take at least $80 \%$ of their prescribed drugs (hazard ratio 1.17) were also associated with a significant increase in the risk of developing XDR-TB. ${ }^{24}$

Demographic and social determinants have also been increasingly identified as a major risk factor for XDR-TB development. In a recent prospective study involving 1,278 patients with MDR-TB from eight countries, women were more likely to have XDR-TB. ${ }^{25}$ Unemployment, alcohol abuse, and smoking were additional risk factors for resistance for second-line drugs in all countries surveyed. In a nationwide MDR survey in Belarus, conducted between 2010 and 2011, history of previous treatment for TB, HIV infection, age less than 35 years, history of imprisonment, disability sufficient to prevent work, alcohol abuse, and smoking were all independent risk factors for MDR-TB. ${ }^{26}$

\section{Prevention}

The best preventive measure to reduce the burden of patients with XDR-TB is appropriate treatment of patients with MDRTB. ${ }^{27}$ Treatment of MDR-TB should adhere to some essential recommendations: use drugs with assured quality, and adopt treatment regimens based on the correct number of drugs, which are given for an appropriate duration.

Early detection and effective treatment of existing patients with XDR-TB is a second pivotal intervention to prevent transmission of XDR-TB strains. Although drug-resistant M. tuberculosis strains may be less fit and thus less infectious, index cases with such organisms are usually infectious for longer periods, and therefore the risk of transmission to their close contacts is higher. ${ }^{9,28}$ Children under 3 years of age who are contacts of patients with MDR-TB or XDR-TB are at a particularly high risk of disease progression; evidence from trials of prophylaxis of XDR-TB contacts are currently unavailable; however, experts suggest that exposed children might benefit from preventive therapy based on the use of two or three oral drugs to which the $M$. tuberculosis strain of the index case is susceptible, given for a minimum of 9 months. ${ }^{9}$
Since there is not a standard preventive regimen with proven efficacy, household and close contacts of patients with XDR-TB should urgently undergo clinical evaluation and follow-up to allow early diagnosis of secondary cases. Although clear evidence is not yet available, the systematic investigation of contacts of known or suspected cases of MDR-TB and XDR-TB may be an effective means of halting the transmission of drug-resistant strains in the community. ${ }^{28}$

Measures to limit transmission through infection-control initiatives are an essential component of any TB program, but are particularly important in settings with high prevalence of MDR-TB and XDR-TB. ${ }^{29}$ Infection-control measures to avoid transmission of XDR-TB strains to household contacts or to contacts within health facilities (other patients and health care workers) do not differ from those recommended to drug-susceptible TB cases. However, cases with MDRTB and XDR-TB may remain contagious for comparatively longer periods before effective treatment stops bacterial replication.

Some studies suggest a reduction in the fitness of resistant M. tuberculosis strains, ${ }^{30}$ but heterogeneity of fitness of these strains has also been described. Thus, new studies are necessary to establish the real infectiousness of XDR-TB strains to contacts under clinical conditions. ${ }^{31}$

\section{Diagnosis}

The definition of XDR-TB relies on two main considerations: the capacity of currently available laboratory tests to reliably detect in vitro resistance of $M$. tuberculosis to rifampicin, isoniazid, fluoroquinolones, and injectable drugs, from one side, and the demonstration that patients bearing XDR-TB strains have a prognosis that differs from that of MDR-TB cases.

Advances were recently made in laboratory methods for detecting MDR-TB. ${ }^{32}$ Automated liquid-culture systems are recommended as the gold standard for secondline DST, which allows identification of XDR-TB in 4-9 weeks. ${ }^{33}$ However, liquid-culture isolation remains labor-intensive, time-consuming, expensive, and requires specialized equipment and a well-serviced biosafety level 3 facility. ${ }^{34}$ As a consequence, in 2012 , only 5\% of new bacteriologically confirmed TB cases underwent DST for first line TB drugs worldwide. ${ }^{3}$ Moreover, DST for both fluoroquinolones and second-line injectable drugs was performed for only $23 \%$ of patients with TB who were confirmed to have MDR-TB, with huge coverage disparities between countries. ${ }^{35}$ 
In order to adhere to current WHO recommendations for second-line DST, which call for testing one fluoroquinolone and all three injectable agents, massive investments are required to strengthen laboratory capacity in the vast majority of settings with high levels of M. tuberculosis drug resistance.

Whenever available, laboratories performing DST for second-line TB drugs should always participate in qualitycontrol programs. The WHO and the International Union Against Tuberculosis and Lung Disease are supporting an international network of supranational laboratories that provide quality control to more than 120 national laboratories. ${ }^{3}$

Once XDR-TB is identified, the optimal choice of drugs to be included in the treatment regimen would require DST of drugs included in group 5 of the TB drug classification. ${ }^{36}$ In one study, early availability of second-line DST results (available within 31 days of treatment initiation) was associated with a significantly better outcome of XDR-TB treatment compared with cases for whom the DST results were available after 31 days of treatment. ${ }^{23}$ However, DST of second-line TB drugs other than quinolones and injectable agents is very poorly standardized, even in supranational reference laboratories, and the capacity of in vitro susceptibility tests to predict clinical efficacy is still undemonstrated for most such drugs. Therefore, treatment of patients with XDR-TB can very rarely be performed on the basis of reliable drugsusceptibility results.

\section{Treatment}

Treatment of XDR-TB cases relies on drugs that are less potent and more toxic than those used in the clinical management of TB disease caused by drug-susceptible or MDR strains., ${ }^{4,37,38}$ A systematic review performed in 2009 on 13 clinical trials showed for the first time that XDR-TB can be successfully treated in up to $65 \%$ of patients, particularly those who are not coinfected with HIV. ${ }^{39}$ However, cases with XDR-TB consistently present in most studies the lowest rates of treatment success and the highest rates of failure, relapse, and death. A more recent meta-analysis covering 560 patients showed a rate of favorable outcomes, defined as either cure or treatment completion, of $43.7 \%$ and a fatality rate of $20.8 \%{ }^{40}$

Recently, Falzon et al analyzed the outcome of almost 7,000 patients from 26 well-established centers with a gradient of resistance from MDR-TB (without additional resistance) to XDR-TB. ${ }^{41}$ The treatment-success rate (compared to treatment failure, relapse, and death) progressively decreased from $64 \%$ for patients with MDR-TB without additional resistance, to $56 \%$ for MDR-TB with additional resistance to second-line injectable agents only, $48 \%$ for MDR-TB with additional resistance to FQs only, and to $40 \%$ for patients with XDR-TB. Remarkably, this poor outcome of $40 \%$ for patients with XDR-TB is very close to the $43.7 \%$ published by Jacobson et al in $2010 .{ }^{40}$

These data highlight one problem of the current XDR definition: patients with XDR-TB may be infected by a strain that is susceptible to other first-line drugs, to a latergeneration FQ, and to other second-line injectable agents, such as capreomycin, and may therefore have a prognosis that is very close to that of MDR-TB.

There is accumulating evidence that the prognosis of patients with XDR-TB is affected by the existence of further resistance to first-line and second-line drugs beyond those included in the definition. Migliori et al recently demonstrated that among patients with XDR-TB (405 in total) the poor prognosis of those without further resistance $(n=301$, treatment success $43 \%$ ) was worsened by the addition of resistance to all second-line injectable agents $(n=68$, treatment success $34 \%$ ), and made still worse by the further addition of resistance to ethambutol and/or pyrazinamide $(\mathrm{n}=42$, treatment success $19 \%) .{ }^{42}$ Interestingly, prognosis was not affected by resistance to any of the group 4 drugs (ethionamide/prothionamide, cycloserine/terizidone, or paraaminosalicylic acid). These data contrast with the observation from a recent meta-analysis on MDR-TB patients, which suggests a better prognosis for patients harboring a strain that was sensitive to ethionamide or prothionamide. ${ }^{43}$ Poor reproducibility of DST for ethionamide may partly explain the inconsistency of findings.

The outcome of XDR-TB treatment may also be positively influenced by the use of drugs that are not widely utilized in a given setting: this seems to be a contributing mechanism to the high treatment-success rates achieved for XDR-TB cases in Peru, where regimens containing drugs such as capreomycin, para-aminosalicylic acid, and cycloserine were used. ${ }^{23,44}$

The optimal number of drugs that should be used in the intensive phase of treatment of XDR-TB is likely to be higher than the four drugs recommended for MDR-TB. ${ }^{37,45-47}$ In the meta-analysis published by Falzon et al, the use of at least six drugs in the intensive phase was associated with better treatment success. ${ }^{41}$ Similarly, the use of at least four effective drugs for patients with XDR-TB and three drugs for MDRTB patients during the continuation phase was associated with the highest odds of treatment success in patients with quinolone-susceptible strains..$^{41,42}$ 
The duration of treatment recommended for MDR-TB seems to be appropriate to treat XDR-TB as well: in the studies published by Falzon et al and Migliori et al, the highest treatment-success rates were observed if the total duration of treatment was extended to $18-20$ or 20-25 months, respectively. ${ }^{41,42}$

There is indirect evidence that treatment of TB is positively affected by the adoption of the best-available standard of care, even in patients with a very extensive pattern of resistance (XDR-TB and beyond). ${ }^{44}$

The role of individual drugs for the treatment of XDRTB cases has not yet been ascertained. Resistance to FQs is part of the definition of XDR-TB strains; however, there is empirical evidence that the use of later-generation FQs significantly improves treatment outcomes of XDR-TB, even when DST demonstrates resistance to a representative FQ. ${ }^{40}$ Incomplete cross-resistance between early generation (ofloxacin) and later-generation (levofloxacin, moxifloxacin) FQs may explain these findings.

There is mounting evidence on the utility of linezolid in managing XDR-TB cases. In a systematic review and meta-analysis on efficacy, safety, and tolerability of linezolid-containing regimes based on individual data analysis of 121 patients from twelve uncontrolled studies, treatment success was achieved in $81.8 \%$ of the cases. ${ }^{48}$ In a randomized trial of patients with XDR-TB randomly assigned to start treatment with linezolid at a dose of $600 \mathrm{mg}$ per day immediately, or after 2 months without a change in their background regimen, culture conversion was achieved in $79 \%$ of patients in the immediate-start group compared with $35 \%$ in the delayed-start group $(P=0.001) .{ }^{49}$ Limitations of linezolid include high cost and toxicity (myelosuppression and neuropathy), which appears to be determined by dose (>600 mg daily) and duration. In the controlled trial reported by Lee et al, of the 38 patients with exposure to linezolid, $31(82 \%)$ had clinically significant adverse events. ${ }^{49}$

Clofazimine is a rediscovered compound in group 5 TB drugs. Its efficacy was shown to be in line with general DR-TB treatment in a systematic review and meta-analysis of twelve evaluable studies of clofazimine-containing regimens. ${ }^{50}$ In addition, clofazimine appears to be associated with a lower incidence of serious adverse effects compared with other second-line drugs. However, the optimal dose of clofazimine and its duration should be better defined by further investigations. ${ }^{50}$

Among antibiotics being proposed for their anti-TB properties, carbapenems show their best performance when combined with clavulanate (generally not available alone, and thus given as an association of high-dose amoxicillinclavulanic acid). A recent case-control study of MDR-TB and XDR-TB cases showed a higher proportion of sputum and culture conversion in patients receiving a standard regimen associated with meropenem clavulanate compared with controls. $^{51}$

Thioridazine, an old neuroleptic drug, has interesting anti-TB properties and an acceptable safety profile. ${ }^{52}$ An uncontrolled study from Argentina showed that the compassionate use of a regime consisting of linezolid, moxifloxacin, and thioridazine produced negative culture conversion in 15 of 17 HIV-negative adult patients with XDR-TB. ${ }^{53}$

New drugs that would help build a better, safer, less toxic, shorter, and cheaper regimen are therefore urgently needed. Three new anti-TB agents presently in the development pipeline (bedaquiline, delamanid, and PA-824) have the potential to enhance our capacity to cure XDR-TB. ${ }^{7,54}$

Bedaquiline is a diarylquinoline (previously known as TMC207) that was granted accelerated approval for the treatment of TB by the US Food and Drug Administration in December 2012, based on data generated by Phase IIb trials. This is the first new anti-TB drug released in more than 40 years and approved specifically for TB. The WHO recommends that bedaquiline may be added to a WHOrecommended regimen in adult MDR-TB patients when there is documented evidence of resistance to any FQ in addition to multidrug resistance, ie, a very close proxy of XDR-TB. ${ }^{55}$ The recommendation is based on a very low quality of evidence, as results from Phase III trials are still awaited. Published data show promising activity on both resistant and susceptible TB; however, concern is still present regarding the safety of the drug, since a higher mortality rate was detected in the group treated with bedaquiline compared with placebo. ${ }^{56,57}$

Delamanid is another very promising compound entering Phase III clinical trials. Used in combination with a background regimen developed according to the WHO guidelines, it showed an increased sputum culture-conversion rate at 2 months in patients with MDR-TB. ${ }^{58}$ In another study reporting the effects of delamanid in combination with an optimized background regimen, a higher proportion of favorable outcomes was observed in the subset of patients with XDR-TB after extended treatment with delamanid (61.4\%) compared with patients receiving the drug for shorter periods $(50 \%) .{ }^{59}$ Notably, all 44 patients with XDR-TB who had received delamanid for at least 6 months survived.

One recent publication on the new anti-TB compound PA-824 shows the potential of a novel approach 
to TB treatment. Outstanding data on the 14-day early bactericidal activity of a three-drug regimen of PA-824, moxifloxacin, and pyrazinamide were reported, ${ }^{60}$ opening the way to a potential universal regimen that would be equally effective against fully susceptible TB and MDR strains.

A public health, program-oriented approach is important to ensure the highest possible success rates for the treatment of XDR-TB. Evidence from Peru suggests that when care is centered at specialized reference sites that adopt strict and well-defined rules, high success rates can be obtained in community MDR-TB programs without hospital care..$^{23,44}$ The outcome of XDR-TB treatment may be improved by strategies to enhance adherence, as well as psychological, nutritional, and financial support, even to a greater extent than that observed for the treatment of drug-sensitive TB. ${ }^{23,44}$

Adverse treatment outcomes for cases with XDR-TB occur more frequently than for other cases of TB with lower levels of drug resistance. ${ }^{39}$ However, most published studies that reported safety data show that the rate and nature of adverse events during XDR-TB treatment is not remarkably different from that of MDR-TB treatment, and suggest that adverse events are not the most important limiting factor for the treatment of XDR-TB. ${ }^{61-63}$

Surgery, an old approach to TB treatment introduced well before anti-TB drugs were made available, gained new importance with the appearance of virtually untreatable XDR-TB cases. ${ }^{64}$ Randomized controlled trials evaluating the efficacy of adjunctive surgical therapy for the treatment of TB have never been reported. However, a systematic review and meta-analysis of pulmonary resection for MDR-TB patients in 15 clinical studies, with a mean of 63 patients per report, showed an estimated pooled treatment-success rate of $84 \%$ (95\% confidence interval 78\%-89\%). ${ }^{65}$ Results of cohort studies comparing treatment outcomes of patients affected by drug-resistant TB being prescribed drug treatment alone versus drug treatment plus surgical resection have been published, though none of these was randomized, so that selection bias remains possible (eg, patients with less severe diseases being included in the surgery arm). ${ }^{66}$ In five of eight studies (all retrospective), patients undergoing surgical resection achieved significantly improved outcomes after adjusting for potential confounders. ${ }^{66}$ In summary, despite encouraging data, the role of surgery remains controversial, as stated in the most recently published MDR-TB treatment guidelines. ${ }^{37,67}$

Relapse rates were similar in MDR-TB and XDR-TB cases that had successfully completed their treatment course in an 8-year follow-up assessment of the Estonia national
MDR-TB program. Although the treatment-success rate was confirmed to be lower among patients with XDR-TB (53.5\% versus $61.1 \%$ ), these data suggest that successful treatment is able to eradicate XDR-TB strains as well. ${ }^{68}$ Among nondrug-related factors, younger age has been shown to be associated with better outcomes in patients with XDR-TB, ${ }^{40}$ as previously demonstrated for drug-susceptible disease. ${ }^{69}$ The existence of comorbidities, such as hypertension or chronic obstructive pulmonary disease, was described as associated with a poor response to treatment or death among patients with XDR-TB. ${ }^{70}$

\section{Conclusion}

XDR-TB may still represent a problem of reduced proportions for most high-burden countries; however, the high morbidity and mortality associated with this form of TB and its potential for expansion warrants high priority being given to this problem and necessary resources being provided for its control. Early and accurate diagnosis of XDR-TB by means of rapid methods, improved management practices of patients with XDR-TB, and private and public investment to identify new and effective drugs are the key tenets for improving individual patient prognosis, as well as limiting the spread of M. tuberculosis strains with a continuously increasing spectrum of resistance.

\section{Disclosure}

The authors report no conflicts of interest in this work.

\section{References}

1. Caminero JA, Torres A. Controversial topics in tuberculosis. Eur Respir J. 2004;24:895-896.

2. Grzybowski S, Enarson DA. [Results in pulmonary tuberculosis patients under various treatment program conditions]. Bull Int Union Tuberc. 1978;53:70-75. French.

3. World Health Organization. Global Tuberculosis Report 2013. Geneva: WHO; 2013. Available from: http://apps.who.int/iris/bitstream/10665/9 1355/1/9789241564656_eng.pdf. Accessed December 2, 2013.

4. Raviglione M, Marais B, Floyd K, et al. Scaling up interventions to achieve global tuberculosis control: progress and new developments. Lancet. 2012;379:1902-1913.

5. Boehme CC, Nabeta P, Hillemann D. Rapid molecular detection of tuberculosis and rifampin resistance. N Engl J Med. 2010;363:1005-1015.

6. World Health Organization. Automated Real-Time Nucleic Acid Amplification Technology for Rapid and Simultaneous Detection of Tuberculosis and Rifampicin Resistance: Xpert MTB/RIF System. Geneva: WHO; 2011. Available from: http://whqlibdoc.who.int/ publications/2011/9789241501545_eng.pdf. Accessed October 25, 2013.

7. Grosset JH, Singer TG, Bishai WR. New drugs for the treatment of tuberculosis: hope and reality. Int J Tuberc Lung Dis. 2012;16:1005-1014.

8. [No authors listed]. Streptomycin treatment of pulmonary tuberculosis: a Medical Research Council investigation. Br Med J. 1948;2:769-782.

9. Zumla A, Abubakar I, Raviglione M, et al. Drug-resistant tuberculosis current dilemmas, unanswered questions, challenges, and priority needs. J Infect Dis. 2012;205 (Suppl 2):S228-S240. 
10. Zumla A, George A, Sharma V, Herbert N, Baroness Masham of Ilton. WHO's 2013 global report on tuberculosis: successes, threats, and opportunities. Lancet. Epub October 22, 2013.

11. World Health Organization. Definitions and Reporting Framework for Tuberculosis - 2013 Revision. Geneva: WHO; 2013.

12. Centers for Disease Control and Prevention (CDC). Emergence of Mycobacterium tuberculosis with extensive resistance to second-line drugs - worldwide, 2000-2004. MMWR Morb Mortal Wkly Rep. 2006;55:301-305.

13. Migliori GB, Besozzi G, Girardi E, et al. Clinical and operational value of the extensively drug-resistant tuberculosis definition. Eur Respir J. 2007;30:623-626.

14. Migliori GB, De Iaco G, Besozzi G, Centis R, Cirillo DM. First tuberculosis cases in Italy resistant to all tested drugs. Euro Surveill. 2007;12:E070517.1.

15. Velayati AA, Masjedi MR, Farnia P, et al. Emergence of new forms of totally drug-resistant tuberculosis bacilli: super extensively drugresistant tuberculosis or totally drug-resistant strains in Iran. Chest 2009; $136: 420-425$.

16. Udwadia ZF, Amale RA, Ajbani KK, Rodrigues C. Totally drugresistant tuberculosis in India. Clin Infect Dis. 2012;54:579-581.

17. Cegielski P, Nunn P, Kurbatova EV, et al. Challenges and controversies in defining totally drug-resistant tuberculosis. Emerg Infect Dis 2012;18:e2.

18. Gandhi NR, Moll A, Sturm AW, et al. Extensively drug-resistant tuberculosis as a cause of death in patients co-infected with tuberculosis and HIV in a rural area of South Africa. Lancet. 2006;368:1575-1580.

19. Dheda K, Shean K, Zumla A, et al. Early treatment outcomes and HIV status of patients with extensively drug-resistant tuberculosis in South Africa: a retrospective cohort study. Lancet. 2010;375:1798-1807.

20. Kvasnovsky CL, Cegielski JP, Erasmus R, Siwisa NO, Thomas K, der Walt ML. Extensively drug-resistant TB in Eastern Cape, South Africa: high mortality in HIV-negative and HIV-positive patients J Acquir Immune Defic Syndr. 2011;57:146-152.

21. Abubakar I, Zignol M, Falzon D, et al. Drug-resistant tuberculosis: time for visionary political leadership. Lancet Infect Dis. 2013;13:529-539.

22. Migliori GB, Sotgiu G, D'Ambrosio 1, et al. TB and MDR/XDR-TB in European Union and European Economic Area countries: managed or mismanaged? Eur Respir J. 2012;39:619-635.

23. Bonilla CA, Crossa A, Jave HO, et al. Management of extensively drug-resistant tuberculosis in Peru: cure is possible. PLoS One. 2008;3:e2957.

24. Shin SS, Keshavjee S, Gelmanova IY, et al. Development of extensively drug resistant tuberculosis during multidrug-resistant tuberculosis treatment. Am J Respir Crit Care Med. 2010;182:426-432.

25. Dalton T, Cegielski P, Akksilp S, et al. Prevalence of and risk factors for resistance to second-line drugs in people with multidrug-resistant tuberculosis in eight countries: a prospective cohort study. Lancet 2012;380:1406-1417.

26. Skrahina A, Hurevich H, Zalutskaya A, et al. Multidrug-resistant tuberculosis in Belarus: the size of the problem and associated risk factors. Bull World Health Organ. 2013;91:36-45.

27. Basu S, Friedland GH, Medlock J, et al. Averting epidemics of extensively drug-resistant tuberculosis. Proc Natl Acad Sci U S A. 2009; 106:7672-7677.

28. World Health Organization. Multidrug and Extensively Drug-Resistant TB (M/XDR-TB): 2010 Global Report on Surveillance and Response. Geneva: WHO; 2010. Available from: http://whqlibdoc.who.int/ publications/2010/9789241599191_eng.pdf. Accessed October 25, 2013.

29. Sotgiu G, D'Ambrosio L, Centis R, et al. TB and M/XDR-TB infection control in European TB reference centres: the Achilles' heel? Eur Respir J. 2011;38:1221-1223.

30. Gagneux S, Long CD, Small PM, Van T, Schoolnik GK, Bohannan BJ. The competitive cost of antibiotic resistance in Mycobacterium tuberculosis. Science. 2006;312:1944-1946.
31. Borrell S, Gagneux S. Infectiousness, reproductive fitness and evolution of drug-resistant Mycobacterium tuberculosis. Int J Tuberc Lung Dis. 2009; 12:1456-1466.

32. McNerney R, Maeurer M, Abubakar I, et al. Tuberculosis diagnostics and biomarkers: needs, challenges, recent advances, and opportunities. J Infect Dis. 2012;205 (Suppl 2):S147-S158.

33. World Health Organization. Policy Guidance on Drug-Susceptibility Testing (DST) of Second-Line Antituberculosis Drugs. Geneva: WHO; 2008. Available from: http://whqlibdoc.who.int/hq/2008/WHO_HTM_ TB_2008.392_eng.pdf. Accessed October 25, 2013.

34. O'Grady J, Maeurer M, Mwabab P, et al. New and improved diagnostics for detection of drug-resistant pulmonary tuberculosis. Curr Opin Pulm Med. 2011;17:134-141.

35. Glaziou P, Falzon D, Floyd K, Raviglione M. Global epidemiology of tuberculosis. Semin Respir Crit Med. 2013;34:3-16.

36. Aziz MA, Wright A, Laszlo A, et al. Epidemiology of antituberculosis drug resistance (the Global Project on Anti-tuberculosis Drug Resistance Surveillance): an updated analysis. Lancet. 2006;368:2142-2154.

37. World Health Organization. Guidelines for the Programmatic Management of Drug-Resistant Tuberculosis: 2011 Update. Geneva: WHO; 2011.

38. Caminero JA, Sotgiu G, Zumla A, Migliori GB. Best drug treatment for multidrug-resistant and extensively drug-resistant tuberculosis. Lancet Infect Dis. 2010;10:621-629.

39. Sotgiu G, Ferrara G, Matteelli A, et al. Epidemiology and clinical management of XDR-TB: a systematic review by TBNET. Eur Respir J. 2009;33:871-881.

40. Jacobson KR, Tierney DB, Jeon CY, Mitnick CD, Murray MB. Treatment outcomes among patients with extensively drug-resistant tuberculosis: systematic review and meta-analysis. Clin Infect Dis. 2010;51:6-14.

41. Falzon D, Gandhi N, Migliori GB, et al. Resistance to fluoroquinolones and second-line injectable drugs: impact on MDR-TB outcomes. Eur Respir J. 2013;42:156-168.

42. Migliori GB, Sotgiu G, Gandhi NR, et al. Drug resistance beyond XDR-TB: results from a large individual patient data meta-analysis. Eur Respir J. 2013;42:169-179.

43. Ahuja SD, Ashkin D, Avendano M, et al. Multidrug resistant pulmonary tuberculosis treatment regimens and patient outcomes: an individual patient data meta-analysis of 9,153 patients. PloS Med. 2012;9:e1001300.

44. Mitnick CD, Shin SS, Seung KJ, et al. Comprehensive treatment of extensively drug-resistant tuberculosis. N Engl J Med. 2008;359:563-574.

45. Orenstein EW, Basu S, Shah NS, et al. Treatment outcome among patients with multidrug-resistant tuberculosis: systematic review and meta-analysis. Lancet Infect Dis. 2009;9:153-161.

46. Johnston JC, Shahidi NC, Sadatsafavi M, FitzGerald JM. Treatment outcomes of multidrug-resistant tuberculosis: a systematic review and meta-analysis. PLoS One. 2009;4:e6914.

47. Falzon D, Jaramillo E, Schünemann HJ, et al. WHO guidelines for the programmatic management of drug-resistant tuberculosis: 2011 update. Eur Respir J. 2011;38:516-528.

48. Sotgiu G, Centis R, D'Ambrosio L, et al. Efficacy, safety and tolerability of linezolid containing regimens in treating MDR-TB and XDR-TB: systematic review and meta-analysis. Eur Respir J. 2012;40: $1430-1442$

49. Lee M, Lee J, Carrol MW, et al. Linezolid for treatment of chronic extensively drug-resistant tuberculosis. $N$ Engl J Med. 2012;367: $1508-1518$.

50. Dey T, Brigden G, Cox H, Shubber Z, Cooke G, Ford N. Outcomes of clofazimine for the treatment of drug-resistant tuberculosis: a systematic review and meta-analysis. J Antimicrob Chemother. 2013;68:284-293

51. De Lorenzo S, Alffenaar JW, Sotgiu G, et al. Efficacy and safety of meropenem/clavulanate added to linezolid containing regimens in the treatment of M/XDR-TB. Eur Respir J. 2013;41:1386-1392.

52. Amaral L. Thioridazine: an old neuroleptic effective against totally drug resistant tuberculosis. Acta Med Port. 2012;25:118-121. 
53. Abbate E, Vescovo M, Natiello M, et al. Successful alternative treatment of extensively drug-resistant tuberculosis in Argentina with a combination of linezolid, moxifloxacin and thioridazine. J Antimicrob Chemother. 2012;67:473-477.

54. Lienhardt C, Raviglione M, Spigelman M, et al. New drugs for the treatment of tuberculosis: needs, challenges, promise, and prospects for the future. J Infect Dis. 2012;205 Suppl 2:S241-S249.

55. World Health Organization. The Use of Bedaquiline in the Treatment of Multidrug-Resistant Tuberculosis: Interim Policy Guidance. Geneva: WHO; 2013.

56. Diacon AH, Pym A, Grobusch M, et al. The diarylquinoline TMC207 for multidrug-resistant tuberculosis. $N$ Engl J Med. 2009;360:2397-2405.

57. Diacon AH, Donald PR, Pym A, et al. Randomized pilot trial of eight weeks of bedaquiline (TMC207) treatment for multidrug-resistant tuberculosis: long-term outcome, tolerability, and effect on emergence of drug resistance. Antimicrob Agents Chemother. 2012;56:3271-3276.

58. Gler MT, Skripconoka V, Sanchez-Garavito E, et al. Delamanid for multidrugresistant pulmonary tuberculosis. $N$ Engl J Med. 2012;366:2151-2160.

59. Skripconoka V, Danilovits M, Pehme L, et al. Delamanid improves outcomes and reduces mortality for multidrug-resistant tuberculosis. Eur Respir J. 2013;41:1393-1400.

60. Diacon AH, Dawson R, von Groote-Bidlingmaier F, et al. 14-Day bactericidal activity of PA-824, bedaquiline, pyrazinamide, and moxifloxacin combinations: a randomised trial. Lancet. 2012;380:986-993.

61. Kim HR, Hwang SS, Kim HJ, et al. Impact of extensive drug resistance on treatment outcomes in non-HIV infected patients with multidrugresistant tuberculosis. Clin Infect Dis. 2007;45:1290-1295.
62. Eker B, Ortmann J, Migliori GB, et al. Multidrug- and extensively drug-resistant tuberculosis, Germany. Emerg Infect Dis. 2008;14: 1700-1706.

63. Keshavjee S, Gelmanova IY, Farmer PE, et al. Treatment of extensively drug-resistant tuberculosis in Tomsk, Russia: a retrospective cohort study. Lancet. 2008;372:1403-1409.

64. Pontali E, Matteelli A, D’Ambrosio L, Centis R, Migliori GB. Rediscovering high technology from the past: thoracic surgery is back on track for multidrug-resistant tuberculosis. Expert Rev Anti Infect Ther. 2012;10:1109-1115.

65. Xu HB, Jiang RH, Li L. Pulmonary resection for patients with multidrug-resistant tuberculosis: systematic review and meta-analysis. $J$ Antimicrob Chemother. 2011;66:1687-1695.

66. Kempker RR, Vashakidze S, Solomonia N, Dzidzikashvili N, Blumberg HM. Surgical treatment of drug-resistant tuberculosis. Lancet Infect Dis. 2012;12:157-166.

67. Blumberg HM, Burman WJ, Chaisson RE, et al. American Thoracic Society/Centers for Disease Control and Prevention/Infectious Diseases Society of America: treatment of tuberculosis. Am J Respir Crit Care Med. 2003;167:603-662.

68. Bloland K, Viiklepp P, Gudmundsson LJ, Altraia A. Predictors of recurrence of multidrug-resistant and extensively drug-resistant tuberculosis. Int J Tuberc Lung Dis. 2012;16:12128-12133.

69. Rieder HL, Cauthen GM, Comstock GW, Snider DE. Epidemiology of tuberculosis in the United States. Epidemiol Rev. 1989;11:79-98.

70. Liu CH, Li L, Chen Z, et al. Characteristics and treatment outcomes of patients with MDR and XDR tuberculosis in a TB referral hospital in Beijing: a 13-year experience. PLoS One. 2011;6:e19399.
Clinical Epidemiology

\section{Publish your work in this journal}

Clinical Epidemiology is an international, peer-reviewed, open access journal focusing on disease and drug epidemiology, identification of risk factors and screening procedures to develop optimal preventative initiatives and programs. Specific topics include: diagnosis, prognosis, treatment, screening, prevention, risk factor modification, systematic

\section{Dovepress}

reviews, risk \& safety of medical interventions, epidemiology \& biostatical methods, evaluation of guidelines, translational medicine, health policies \& economic evaluations. The manuscript management system is completely online and includes a very quick and fair peer-review system, which is all easy to use. 